\title{
Research of the elasticity of electric energy tariff demand on competitive market of unified energy system of the Ural
}

\author{
V.G. Mokhov ${ }^{1, *}$, and T.S. Demyanenko ${ }^{1}$ \\ ${ }^{1}$ South Ural State University, Chelyabinsk, Russia
}

\begin{abstract}
The aim of the study is to analyze the youngest in the world competitive market of electric energy and power of Russia. The hypothesis that there is a significant time lag between the launch date regulated competitive energy market and the actual state of its transition to a stationary operation. To test the hypothesis covered the electric energy consumer's reaction on tariffs changes in the UES of the Ural in the period of time after restructuring RJSC "UES of Russian Federation". Factual data of UES of Russia's System operator shows how indicator values of electric energy consumption elasticity changes: consuming turns from elastic to inelastic according to price. Proved that in 2014 the energy demand matches to a competitive market. As a methodical research tool used 5 types of regression analysis equations for time series of electricity consumption, in addiction by the rate of the day-ahead market for 2009-2014. The research found that the transition to a competitive market of electricity production in Russia in fact was didn't carried out in 2008, but was carried in 2014. Research proved that the electricity market regression analysis applied in predicting short periods of time (day-ahead market), because by increasing the time interval of forecasting accuracy of the forecast is decreases. The research results have great practical significance for the power industry subjects, especially those with high energy intensity of production, as the increase in the accuracy of forecasting reduces fines and total costs for electricity.
\end{abstract}

\section{Introduction}

Electric energy is one of the most important products of intrabrach consumption and constitutes the considerable share of the expenditures practically in all branches of economy. As the electrical energy industry is the leading infrastructure branch, it is noncontestantly defines the limitation of economy's development. This calls for outrunning growth of generating capacities as well as deep changes in qualitative characteristics. Electric energy deficiency in some regions (and even in the whole country) inevitably leads to restriction of economic growth [1].

In today's existing view the Wholesale Power Market (WPM) is functioning from September 1, 2006, when the decree of the Government of the Russian Federation introduced new rules for the functioning of the wholesale market. At present, the Russian wholesale electricity market is the youngest and least explored in the world.

During operation the Wholesale Power Market accumulate historical values of economical and physical parameters in database and this drastically increases the volumes of input information for forecasting tasks of electricity. Also hardware and software development facilitated the creation of powerful computing platforms with which the implementation of the difficult forecasting algorithms is possible. In the meantime modern approaches to economic and technical management lodge tough requirements to the accuracy of predictions [2]. As the result, the task of forecasting the time sequences becomes harder with development of informational technologies.

The aim of this research is to determine the type of correlation between the amount of electric energy consumption and the rate of the day-ahead market and the definition of the elasticity of demand for electricity by the rate on the basis of the analysis which we can be seen on the construction of a competitive market.

Based on the goal implies following the research objectives:

- construction 5 types of regression equations (linear, hyperbolic, power, exponential and reverse) to forecast the consumption of electric energy;

- evaluation of forecast errors in forecasting consumption by derived regression equation;

- determination the coefficient of elasticity of demand by the rate for obtained each regression model.

Relevance assigned tasks is obvious, because by the lack of precision forecasting model energy consumers are losing their competitive edge, what leads to deterioration of financial and economic indicators of the subjects of the electricity market and even crises. Electricity market officially became competitive in 2006, but the transition from a monopoly to a competitive pricing can actually display the elasticity coefficient, which shows whether the increase or decrease in tariffs affect the demand [3].

*Corresponding author: mokhov50@mail.ru 


\section{Theoretical Aspects}

Time sequence is a sequence of time ordered index numbers, which characterize the state and level of changing of the event in question [4].

The source elaborates the etymology of time sequence and how statistics of Russia is unaccustomed to this term unlike statistic series and statistic research of dynamics terms [5].

Now there are a lot of methods for time-sequences prediction. They can be divided into intuitive and formalized [6]. Formalized methods are especially interesting because of high accuracy rate.

Quite often in literature two close groups of methods are classified as one group, as it is described in the source [7]. Formalized methods of prediction are methods, which use mathematic description of revealed trends in objects development for acquiring a prognosis. Factographic methods use realities (or fait accompli) already accomplished fact, which are recorded one any storage medium with the help of quantitative or qualitative characteristics as the source of information. In reality both of these groups use one and the same methods of prediction and that's why they are equated so often and classified in one group [8].

Formalized methods are divided into statistic prediction methods, imitational forecasting, historiclogical analysis, analogies methods and advancing methods [9].

Statistic methods define certain equation, which was acquired on the basis of previously researched timepredicted value of the time-series. This equation reflects the correlation between future sequence values and its history and/or external factors [10]. The last three methods are sometimes grouped under the name of "artificial intelligence methods": they imitate the process of human's reasoning in the studying of history or predicting future sequence values.

Methods of statistic analysis, which are good for defining functional dependences in electric energy industry are quite various in their nature. This research will bring light to the method of correlational regressional analysis.

\section{Input data and research methods}

The essence of method of correlational regressional analysis is in the following:

1) In building and analysis of statistic model as a regression equation (equation of correlation connection) that approximately expresses the dependence of resultative attribute $(y)$ from one or more factors $(x)$.

2) In evaluation of tightness and defining the type of correlation between the resultative attribute and factors exerting influence on it [11].

In our case the task is to compose an equation of correlation (regression equation), which would define the correlation between the electrical energy tariffs $(x)$ and the level of consumption $(y)$, as well as in analysis of tightness between these factors [12].
Let's build the field of set-up attributes correlation. Input data is taken from the official site of System operator of the Unified Power System of Russia (www.http://so-ups.ru/). For calculations the following data were gathered: consuming data and market prices for the 24 hours ahead (Day Ahead Market) on Unified Power System (UPS) of the Ural from 01.01.3013 till 31.12.2013.

The type of correlation connection cannot be defined according to the field of correlation (Fig. 1). Moreover, it can be assumed that the correlation connection between the attributes is virtually nonexistent. However, the reduction of the viewed interval (i.e. till January of 2013 (Fig. 2)) extracts the obvious linear correlation. Therefore, before assuming the existence of the correlation the elasticity coefficient for different types of models (linear, power, exponential, hyperbolical and negative) must be calculated and the level of connection of factor (independent variable) with the result (dependent variable) must be determined.

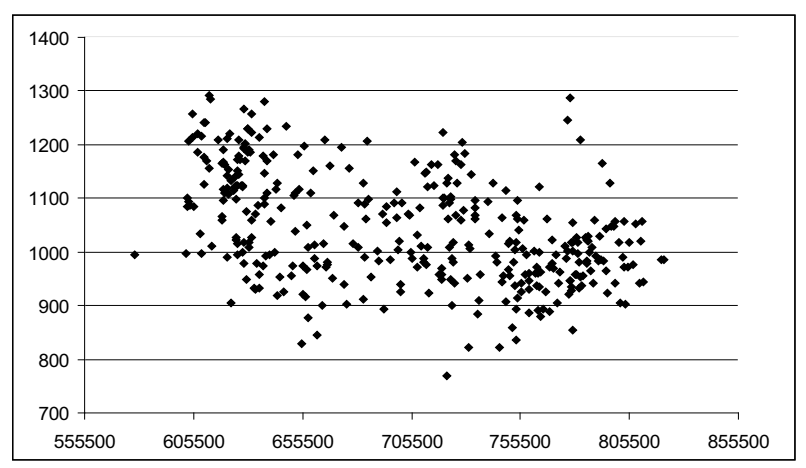

Fig. 1. The field of electric power consumption correlation and DAM tariff for January - December period of 2013 year.

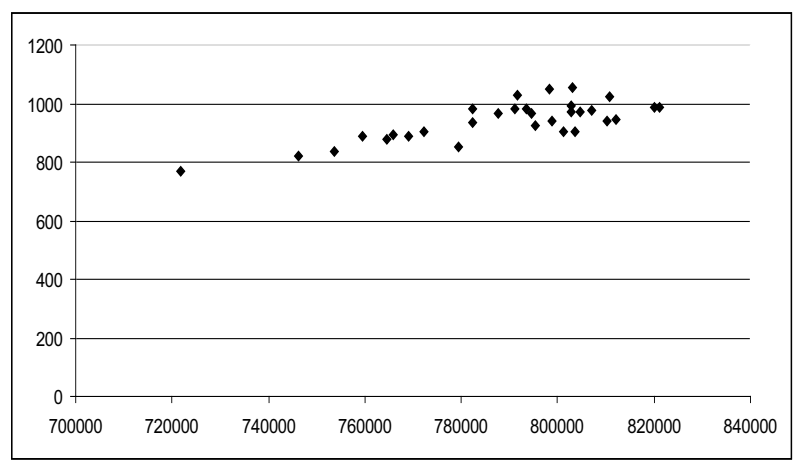

Fig. 2. The field of electric energy consumption and DAM tariff correlation in January 2013.

The second task of this research was to study the dependence between DAM prices and the amount of electric energy consumption. The task of defining the marked neighbouring relationship is in the following: to measure with the help of special values how far correlation connection approaches the actual, according to its force. One of these parameters can be elasticity coefficient [13]. It represents the index of constraint force of factor $\mathrm{x}$ with the result $\mathrm{y}$ and shows 
the percents of increase of $y$ when the value of factor changes by 1 It will be appropriate to define elasticity coefficient for each type of regression.

If elasticity coefficient is less than 1 in absolute value then we can say that the variable $\mathrm{y}$ along the $\mathrm{x}$ is inelastic. If elasticity coefficient is more than 1 then we can say that $\mathrm{y}$ is elastic along the $\mathrm{x}$, because each percent of changing of factor leads to even greater changes of y [14]. If coefficient of elasticity equals 1 it is called unitary elasticity. In the limiting case when coefficient of elasticity equals eternity it is called absolute elasticity. And, accordingly, when the coefficient of elasticity is zero we call it absolute inelasticity [15].

Below are the mathematical models determining the coefficients of regression equations, the coefficient of elasticity of demand at the rate of error detection and time series forecasting.

\section{Mathematical models}

\section{A. Construction of correlation-regression analysis equations}

An equation of linear regression (LR). The resultative attribute is this correlation is changed under the influence of factor attribute, it can be written as:

$$
y_{x}=a x+b,
$$

where $a$ is a coefficient of regression.

To find the theoretical equation of correlation in this case is to define the parameters of a right line. Those parameters can be found with the least-squares method which as the result gives the following system of normal equation for finding the parametres of a right line:

$$
\left\{\begin{array}{l}
a \sum x+n b=\sum y \\
a \sum x^{2}+b \sum x=\sum x y
\end{array}\right.
$$

Solving this equation systems results in finding the desired parameters [16].

$$
\left\{\begin{array}{l}
a=\frac{n \sum x y-\sum x * \sum y}{n \sum x^{2}-\left(\sum x\right)^{2}} \\
b=\frac{\sum x^{2} * \sum y-\sum x * \sum x y}{n \sum x^{2}-\left(\sum x\right)^{2}}
\end{array},\right.
$$

where $a, b$ are the parameters of the regression equation; $y$ is a resultative attribute; $x$ is a factor attribute; $n$ is a total number.

Coefficients of the regression equation can be deduced with the following formulas: $\%$.

$$
a=\frac{\overline{x y}-\bar{x} \cdot \bar{y}}{\overline{x^{2}}-(\bar{x})^{2}}, b=\bar{y}-b \bar{x},
$$

$$
\begin{array}{r}
\bar{x}=\frac{\sum x_{i}}{n}, \quad \bar{y}=\frac{\sum y_{i}}{n}, \quad \overline{x y}=\frac{\sum x_{i} y_{i}}{n}, \\
\overline{x^{2}}=\frac{\sum x_{i}^{2}}{n}, \quad n=365
\end{array}
$$

The equation of power regression (PR) looks like:

$$
y=b_{0} x^{b_{1}}
$$

Let's linearize the model for more convenient way of finding the coefficients of those models and introduce new variables:

$$
v=\ln y, \quad u=\ln x .
$$

As the result we get the linear regression model:

$$
v=a_{0}+a_{1} u, \text { where } a_{0}=\ln b_{0}, \quad a_{1}=b_{1} .
$$

Coefficients of this equation are deduced from these formulas:

$$
a_{1}=\frac{\overline{u v}-\bar{u} \cdot \bar{v}}{\overline{u^{2}}-(\bar{u})^{2}}, a_{0}=\bar{v}-a_{1} \bar{u}
$$

Exponential regression (ER) looks like: $y=b_{0}+b_{1} e^{x}$.

New variables are introduces to linearize the model:

$$
v=y, \quad u=e^{x} .
$$

To make calculations simpler we introduce a multiplier for $x$, for example 0,001 . So the regression equation looks like:

$y=b_{0}+b_{1} e^{0,001 x}$, let's introduce the variables for linearization:

$$
v=y, \quad u=e^{0,001 x}
$$

As the result we get linear regression model:

$$
v=a_{0}+a_{1} u, \text { where } a_{0}=b_{0}, \quad a_{1}=b_{1} .
$$

Hyperbolical regression (HR) looks like:

$$
y=b_{0}+b_{1} \frac{1}{x}
$$

Linearize the given model. To do this we introduce the following variables:

$$
v=y, \quad u=\frac{1}{x} .
$$

As the result we get the linear regression model:

$$
v=a_{0}+a_{1} u, \text { where } a_{0}=b_{0}, a_{1}=b_{1} .
$$

Reverse regression (RR) looks like: $y=\frac{1}{b_{0}+b_{1} x}$.

where 
Let's linearize this model. In order to do this we should introduce new variables:

$$
v=\frac{1}{y}, \quad u=x .
$$

As the result we get linear regression model:

$$
v=a_{0}+a_{1} u, \text { where } a_{0}=b_{0}, \quad a_{1}=b_{1} .
$$

\section{B. Determination of the coefficient of elasticity of demand by the rate}

Elasticity of demand $(W)$ according to tariff $(P)$ is [17]:

$$
E_{W}=\frac{\partial W / W}{\partial P / P}
$$

\section{Determination of the forecast error}

The accuracy of time sequences forecasting can be estimated with the help of mean absolute percentage error (MAPE) [18]:

$$
M A P E=\frac{1}{n} \sum_{i=1}^{n} \frac{|y(i)-\hat{y}(i)|}{y(i)} \cdot 100 \%,
$$

where $y(i)$ - actual value, $\hat{y}(i)$ - predicted value.

\section{Results}

The regression equations, based on the data of 2009-2014 years, were calculated in order to get fuller information about the possibility of applying regression models for predicting the electric energy consumption.

The forecasted values were calculated for each of January next year (forecast for 31 days) and on January, 1 of each of the following year (forecast for 1 day). In addition to the construction of forecast values of electricity consumption was researched elasticity coefficient for the $2009-2014$ years. Summary results are presented in Table 1.

For visualization of the results let plot a graph of actual and forecast values of electricity consumption in January 2014.

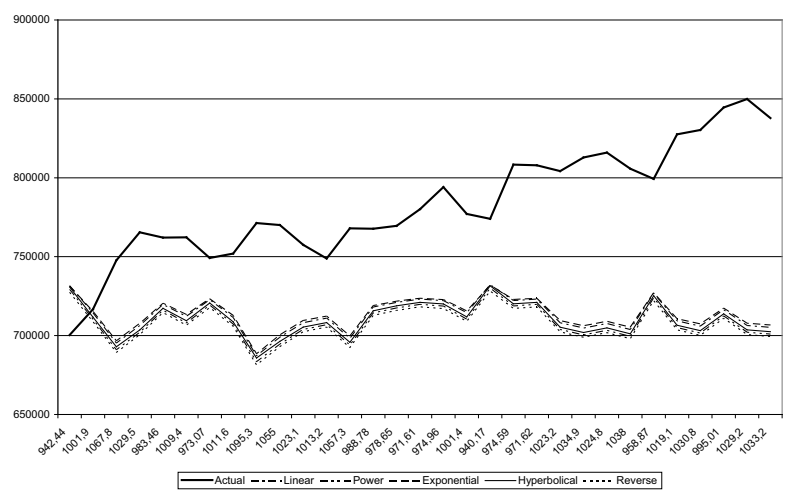

Fig. 3. Actual and predicted values of consumption for regressions built for January 2014.
This diagrams show:

- predicted values, which are obtained by the already found regression equations, are close;

- dynamics of the predicted values is the same;

- with time the amount of forecasting errors increases.

The mean error of the forecast for this period for the regression models in forecasting the first month of each year amounted to $11.06 \%$, and in the forecast of the first day of every year $-4.64 \%$.

On this stage of research the conclusion can be drawn that the most appropriate regression forecasting model of all is the model of exponential regression, because on the average it gives the smallest percent of forecasting error. Its appliance is possible through time sequence prediction.

Its application is possible when forecasting time series at short intervals of time, that is, to predict the parameters of day-ahead market, as the average error for months $-10.44 \%$, and for one day $-4.14 \%$, which is quite satisfactory for the current economic situation.

The main disadvantage of this approach for predicting the amount of electric energy consumption is the fact that the DAM prices values is considered to be fixed. It will never be possible in reality, because in order to predict the amount of consumption you must predict the DAM prices first, using other methods. All this in practice gives even greater percent of forecasting error. Forecasting error for tariffs will add up to the error for amount of consumption [19].

Elasticity coefficient dynamics for each regression model is represented on Fig. 4. From dynamics it is clearly visible that the equations of hyperbolic regression give the highest elasticity coefficient.

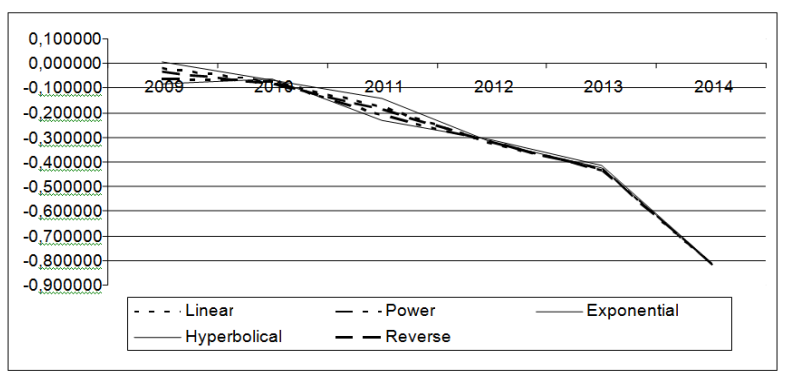

Fig. 4. Elasticity coefficients dynamics for regression models in 2009-2014.

Analysis of the acquired results allows us to draw the following conclusions:

1) The reaction of consumers on changing tariffs for all the analyzed years for all the models is practically the same: with growth of tariff pressure the electric energy consumption becomes smaller, and it is described by the sign «(-) in the indexes of the price demand elasticity.

2) 2009-2011 time period is marked with absolute price inelasticity of demand. It is connected with the beginning of reform of price determination in electrical energy industry.

3) In the period of 2012-2014 the elasticity coefficient gradually increases, but in 2012-2013 its indexes are still a lot lower than 1 , which is the obvious characteristic of inelasticity.

4) In 2014 coefficient of inelasticity reaches the level, when we can talk about tariff elasticity of demand, because if the tariff growth rate is $10 \%$ then the electrical energy demand falls more than on $8 \%$. 
Table 1. Calculation of prediction errors for each type of regression equation when forecasting for 1 day and 1 month for 2009-2014 years.

\begin{tabular}{|c|c|c|c|c|c|}
\hline$\stackrel{\succsim}{\check{\nu}}$ & $\sum_{i=1}^{0}$ & Regression equation & $\begin{array}{l}\text { Day forecasting } \\
\text { error, \% }\end{array}$ & $\begin{array}{l}\text { Month forecasting } \\
\text { error, \% }\end{array}$ & $\begin{array}{l}\text { Elasticity } \\
\text { coefficient }\end{array}$ \\
\hline \multirow{5}{*}{ 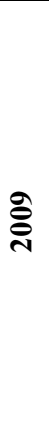 } & $\boldsymbol{\sim}$ & $\hat{y}=-19,822 x+656683,435$ & 14,88 & 7,50 & $-0,02$ \\
\hline & $\sim \simeq$ & $\hat{y}=952642,266 x^{-0,062}$ & 16,29 & 8,04 & $-0,061$ \\
\hline & 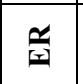 & $\hat{y}=637890,070+3203,832 e^{0,001 x}$ & 14,10 & 7,54 & 0,006 \\
\hline & $\cong$ & $\hat{y}=588503,779+34695770,567 \cdot \frac{1}{x}$ & 16,14 & 7,60 & $-0,084$ \\
\hline & $\stackrel{x}{\simeq}$ & $\hat{y}=\frac{10^{9}}{1518,1432+0,081859 x}$ & 16,22 & 8,53 & $-0,033$ \\
\hline \multirow{3}{*}{ 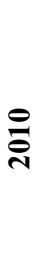 } & $\dddot{a}$ & $\hat{y}=-57,919 x+719514,4345$ & 13,83 & 6,16 & $-0,073$ \\
\hline & $\frac{a}{a}$ & $\hat{y}=1106550,962 x^{-0,075}$ & 14,31 & 6,72 & $-0,075$ \\
\hline & $\underline{\underline{a}}$ & $\hat{y}=726350,995-23828,193 e^{0,001 x}$ & 13,80 & 6,12 & $-0,07$ \\
\hline \multirow{2}{*}{ 을 } & $\cong$ & $\hat{y}=628992,782+34712848,248 \cdot \frac{1}{x}$ & 13,72 & 6,23 & $-0,062$ \\
\hline & $\stackrel{\varkappa}{\simeq}$ & $\hat{y}=\frac{10^{9}}{1382,3267+0,148132 x}$ & 14,84 & 7,17 & $-0,082$ \\
\hline \multirow{5}{*}{$\overline{\bar{\nu}}$} & $\dddot{y}$ & $\hat{y}=-130,020 x+815853,0481$ & 8,61 & 4,70 & $-0,178$ \\
\hline & $\stackrel{a}{a}$ & $\hat{y}=2962106,548 x^{-0,213}$ & 8,69 & 6,17 & $-0,212$ \\
\hline & $\stackrel{\mathscr{r}}{\underline{x}}$ & $\hat{y}=797903,412-40664,163 e^{0,001 x}$ & 8,91 & 3,41 & $-0,143$ \\
\hline & $\cong$ & $\hat{y}=530694,006+152397360,944 \cdot \frac{1}{x}$ & 8,29 & 8,17 & $-0,233$ \\
\hline & $\dddot{\varkappa}$ & $\hat{y}=\frac{10^{9}}{1183,706+0,288532 x}$ & 9,21 & 4,25 & $-0,186$ \\
\hline \multirow{5}{*}{ בֶב } & $\simeq \simeq$ & $\hat{y}=-232,887 x+926583,7874$ & 10,37 & 3,57 & $-0,327$ \\
\hline & $\sim \simeq$ & $\hat{y}=6405542,995 x^{-0,323}$ & 10,91 & 3,89 & $-0,321$ \\
\hline & $\frac{\Omega}{|r|}$ & $\hat{y}=930311,412-86451,264 e^{0,001 x}$ & 10,24 & 3,05 & $-0,323$ \\
\hline & $\cong$ & $\hat{y}=479021,242+211964999,329 \cdot \frac{1}{x}$ & 10,69 & 4,56 & $-0,31$ \\
\hline & $\stackrel{\simeq}{\simeq}$ & $\hat{y}=\frac{10^{9}}{974,2196+0,481210 x}$ & 11,14 & 3,07 & $-0,323$ \\
\hline \multirow{3}{*}{$\stackrel{m}{\stackrel{2}{\nu}}$} & $\simeq$ & $\hat{y}=-289,966 x+1004844,169$ & 9,11 & 4,46 & $-0,431$ \\
\hline & $\sim \simeq$ & $\hat{y}=14033988 x^{-0,432}$ & 9,58 & 4,13 & $-0,429$ \\
\hline & 寽 & $\hat{y}=990942,326-101156,731 e^{0,001 x}$ & 8,98 & 4,43 & $-0,427$ \\
\hline
\end{tabular}




\begin{tabular}{|c|c|c|c|c|c|}
\hline & $\cong$ & $\hat{y}=408526,442+303681877,581 \cdot \frac{1}{x}$ & 9,41 & 4,34 & $-0,414$ \\
\hline & $\cong$ & $\hat{y}=\frac{10^{9}}{803,241+0,606418 x}$ & 9,77 & 3,86 & $-0,437$ \\
\hline \multirow{5}{*}{ 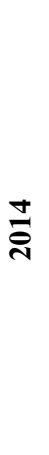 } & $\simeq$ & $\hat{y}=-526,296 x+1293032,026$ & 7,23 & 0,43 & $-0,82$ \\
\hline & $\frac{a}{2}$ & $\hat{y}=231484376,2 x^{-0,826}$ & 8,73 & 0,38 & $-0,821$ \\
\hline & $\stackrel{\mathscr{L}}{\underline{\mathbf{I}}}$ & $\hat{y}=1231325,538-171564,275 e^{0,001 x}$ & 6,60 & 0,27 & $-0,809$ \\
\hline & $\cong$ & $\hat{y}=129780,315+639040284,463 \cdot \frac{1}{x}$ & 8,78 & 0,56 & $-0,813$ \\
\hline & $\stackrel{\mathscr{x}}{\underline{\underline{\alpha}}}$ & $\hat{y}=\frac{10^{9}}{242,5855+1,063704 x}$ & 8,61 & 0,14 & $-0,822$ \\
\hline
\end{tabular}

Averaged elasticity coefficient for 2014 was around $-0,82$, so, we can talk about tariff elasticity amount consumption. It can be seen in Fig. 5. When the price increases for $10 \%$, the amount of consumption decreases for $8,2 \%$.

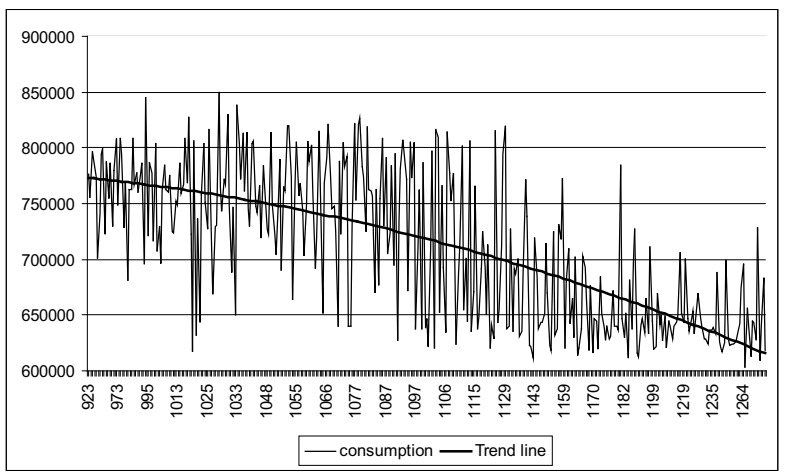

Fig. 5. Dependence of the consumption amounts in the electric energy tariffs in 2014.

Experience of many years in the field of evaluation of electric energy demand functions allowed G. Kouris [20] to draw conclusions even in 1981, which are still solid in our days:

- evaluation of elasticity coefficients crucially depends on the existence of data, model and structure of economy specifications at each moment of time;

- the term "absolute elasticity" is more of an illusion, than reality;

- elasticity coefficients change with time under cause and effect relations influence;

- electric energy demand predictions should be based on statistically evaluated elasticities and not on predicted ones [21].

\section{Conclusions}

1) The research was based on the data of 2009-2014 years on prices and amounts of electric Energy consumption in CPS of the Ural region, acquired from official cite of System Operator of United Power System of Russia.
2) 5 types of regression models were built (linear, power, exponential, hyperbolical, reverse) for each year under consideration.

3) On the basis of these models predicted values of consumption amounts were calculated for all January and only 1 January of the year, following the researched one. The average predicting error of applying regression models amounted in $11 \%$ and $4,64 \%$ respectively. The lowest forecast error showed exponential regression model in predicting short time intervals $-4.14 \%$.

4) Restructuring of RJSC "UES of Russian Federation was introduced in 2008, but the transition to competitive market of electric energy manufacturing didn't happened. The electric energy demand gets closer to tariff elasticity only in 2014, and it is depicted in elasticity coefficient 0,82 .

5) In 2014 the trend appeared that portrayed the dependence of consumption volume of electricity from the electric energy tariff.

6) It is proved that the electricity market for the regression analysis applied in predicting short periods of time, or for the day-ahead market, as by increasing the time interval of forecasting accuracy of the forecast is reduced.

7) The Market of electric energy and power in Russia of monopoly, when the manufacturer has determined the tariffs, in 2014 became competitive.

The practical significance of the results of the study is that in 2014 electricity producers can no longer dictate the tariffs, and must build up its economic activities to the market behavior of energy consumers.

The work was supported by Act 211 Government of the Russian Federation, contract № 02.A03.21.0011.

\section{References}

1. P.F. Drucker, Management challenges for the $21 \mathrm{st}$ century (HarperBusiness, New York, 1999)

2. D. Salvatore, Microeconomics: theory and applications (Oxford University Press, Inc, 2003) 
3. F.J. Nogales, A.J. Conejo, Journal of the Operational Research Society, 57, 4, 350-356 (2006)

4. V.N. Afanasyev, M.M. Yuzbashev, Time sequences analysis and forecasting (Finance and statistics; INFRA-M, Moscow, 2012)

5. E.E. Tikhonov, Forecasting in conditions of the market (Nevinnimyssk, 2006)

6. M. Norizan, A. Maizah Hura, I. Zuhaimy, Proc. Regional Conference on Statistical Sciences, 5773 (2010)

7. M.M. Butakova, Economics and forecasting. Methods and ways of practical calculations (KNORUS, Moscow, 2008)

8. M. Beenstock, A. Dalziel, Energy Economics, 4, 90-98 (1986)

9. R. Haas, L. Shipper, Energy Economics, 20, 421442 (1998)

10. H.K. Alfares, M. Nazeeruddin, International Journal of Systems Science, 33, 23-34 (2002)

11. L. Hunt, G. Judge, Y. Ninomiya, Energy Economics, 25, 93-118 (2003)

12. Dj. Box, G.M. Djenkins, Time series analysis, forecasting and management (Mir, Moscow, 1974)
13. R. Nesbakken, Energy Economics, 21, 493-515 (1999)

14. I.A. Bashmakov, Evaluation of price elasticity of electric energy demand parameters for different groups of consumers and territorial entities of Russian Federation (TSENEF, Moscow, 2007)

15. S. Singh, Cybernetics and SystemsAnInternational Journal, 31, 49-65 (2009).

16. V.G. Mokhov, Proc. Science SUSU, 2, 83-86 (2009).

17. V.L. Jirnov, Fundamentals of energy pricing: Lectures (Publishing center NSTU, Novosibirsk, 1999)

18. P.A. Sinutin, Formation of system of state regulation of the energy supply in the Russian Federation (Economic, Moscow, 2002)

19. V.G. Mokov, T.S. Demyanenko, Science SUSU. Section of Economics and management, 2, 83-86 (2009)

20. G. Kouris, Energy Economics, 5, 89-99 (1983)

21. J.W. Taylor, P.E. McSharry, IEEE Transactions on Power Systems, 22, 2213-2219 (2008) 